\title{
Productivity Dynamics and Structural Change in the U.S. Manufacturing Sector
}

\author{
by \\ Jens J. Krüger ${ }^{1}$ \\ Friedrich-Schiller-University Jena \\ February 2005
}

Department of Economics, Carl-Zeiss-Strasse 3, D-07743 Jena, Germany, Tel.: +49 3641943 203, Fax: +49 3641943 202, E-Mail: jens.krueger@wiwi.uni-jena.de

\begin{abstract}
:
The paper investigates structural change among the four-digit (SIC) industries of the U.S. manufacturing sector during 1958-96 within a distribution dynamics framework. Focus is on the transition density of the Markov process that characterizes the value added shares of the industries. This transition density is estimated nonparametrically as well as by maximum likelihood, in which case the functional form of the density is derived from a search theoretic model. The nonparametric and the maximum likelihood fits show striking similarities. The relation of structural change to a relative measure of total factor productivity change is tested by an application of quantile regression and is found to be significantly positive throughout.
\end{abstract}

JEL classification: L16, O30, L60, C14

Keywords: structural change, productivity, manufacturing, quantile regression

--- The paper is not competing to the Young Economist Award ---

\footnotetext{
I would like to thank Uwe Cantner and Peter Kischka for helpful suggestions. The remaining errors are all in my personal responsibility.
} 


\section{Introduction}

Structural change in the economy implies that some sectors or industries grow faster than others in the long-run. This pattern is associated with shifts of the shares that these sectors or industries have in the total. Well established are the long-run shifts of the shares of the three main sectors of the private economy: agriculture, manufacturing and services (see e.g. Kuznets (1966)). These shifts are to a large extent caused by demand-side changes due to different income elasticities for agricultural, manufacturing and service goods. Kongsamut et al. (2001) analyze a three-sector general equilibrium growth model with a common rate of (exogenous) technological progress and nonhomothetic consumer preferences to explain the pattern of declining agriculture and manufacturing shares and a rising service share.

In addition to this, supply-side changes caused by technological progress also play an important role in the process of structural change. Baumol (1967) provides a theoretical explanation for the shift of employment from manufacturing to services based on different rates of technological progress in these sectors. Baily et al. (1996) give a comprehensive descriptive empirical account of the relation of (labor) productivity change and plant employment changes in U.S. manufacturing during 1977-87. Their results disagree with the conventional view that productivity improvements during these years are systematically associated with downsizing in terms of plant employment. They emphasize the large role of idiosyncratic factors as does the comprehensive survey of Bartelsman and Doms (2000). Harberger (1998) reviews research on productivity growth at both the firm and sectoral level and also finds much dispersion in the productivity developments.

The present paper aims at expanding the research on structural change by taking a closer look at the relation of total factor productivity growth and changes of the real value added shares of industries within the U.S. manufacturing sector. Hence we are interested in intra-sectoral structural change. The analysis is performed in a distribution dynamics framework with focus on the investigation of the Markov process that governs the dynamics of the distribution of the the value added shares. A theoretical model based on search theoretic considerations is developed to motivate the interrelation of share dynamics and differential productivity development in the distribution dynamics context. This model provides a specific representation of the 
stochastic transition law of the Markov process in form of the transition density which is subsequently estimated by maximum likelihood. In a last part, the relation of productivity and structural change is statistically tested using the approach of quantile regression which permits to uncover differential effects of productivity growth at different positions of the support of the distribution of the value added shares. The empirical analysis is carried out for U.S. manufacturing industries on the detailed four-digit (SIC) level of aggregation over the period 1958-96.

From a methodological point of view, the distribution dynamics framework applied here is particularly suited to the specific requirements of the analysis of structural change. This approach allows to deal with the complexity of structural change by summarizing the differential developments of industries in a way that makes the heterogeneity within an aggregate and the change of this heterogeneity visible without having to consider each industry separately. In the economic literature the distribution dynamics approach has been applied to analyze the dynamics of the world income distribution by Quah (1996a,b), in the theoretical modelling of intergenerational changes of the income distribution by Loury (1981) as well as in the research that investigates the firm-size distribution and the validity of Gibrat's law as summarized by Sutton (1997).

The results obtained with this approach show that the Markov process that governs the value added share distribution is associated with a roughly stationary distribution and with a transition density that shows that structural change is present as a long-run process. These features can be replicated by a theoretical model which leads to a transition density that, when estimated by maximum likelihood, looks very similar to the nonparametric kernel estimate. The intervening role of technological change in the form of productivity growth postulated by the model is confirmed by the quantile regression estimates. These results show that structural change in the U.S. manufacturing sector is systematically influenced by technological change.

The plan of the paper is as follows: Following this introduction section 2 contains an empirical exploration of the distribution of the value added shares of the U.S. manufacturing industries and the change of this distribution over time. This leads to the identification of certain critical aspects that the theoretical model introduced in section 3 is intended to explain. This 
model is constructed around a Markov process for the value added shares which is affected by the productivity growth of the industries relative to each other. A search mechanism is introduced to derive the transition kernel of the Markov process from the distribution of productivity change via the distribution of the largest order statistic. In section 4, the transition density associated with this kernel is transformed and estimated by maximum likelihood. Following the description of the (again nonparametric) procedure to measure productivity change and the data used for this task in section 5 we proceed in section 6 with a brief description and the application of the method of quantile regression to test the relation of productivity growth and structural change postulated in the model. Section 7 summarizes the main findings and concludes.

\section{Exploration of Structural Change}

The structural composition of the U.S. manufacturing sector is quantified in this work by the shares of the four-digit industries in total real value added of the manufacturing sector. The value added shares are used here to represent the relative importance of the single industries within the manufacturing sector. Compared to employment, real value added of industries is more oriented at the contribution to economy-wide GDP and probably less affected by changing regulations (e.g. of the labor market). The data are taken from the NBER-CES manufacturing industry database which covers the period 1958-96 for more than 450 four-digit (SIC) industries and is described in Bartelsman and Gray (1996). Real value added for each industry and year is computed as the ratio of the data series for the value added [VADD] and the price deflator of the value of shipments [PISHIP]. ${ }^{2}$ This real value added variable is subsequently divided by the total real value added of the whole manufacturing sector in the respective year to reach the shares of the four-digit industries in total real value added of the manufacturing sector.

Explored in the context of the distribution dynamics approach are the changes of the shape of the density function of these value added shares and the stochastic transition law that

\footnotetext{
2 The abbreviations in parentheses are those of the appendix of Bartelsman and Gray (1996). The alternative way to calculate real value added by subtracting the real costs of energy and other materials from the real value of shipments leads to results that are very similar to those reported here.
} 
visualizes the intra-distributional changes in the form of the transition density. The latter as a conditional object gives a complete probabilistic account of the possbile transitions and reveals much more about the data generating process than does the shape of the distribution as an unconditional object alone (see Brock (1999) and Quah (1996a)). All density functions are estimated by kernel methods with Gaussian kernels after logging the data to avoid boundary biases of the kernel estimator (see Wand and Jones (1995)).

To estimate the shape of the density by the kernel density estimator the bandwidth parameter is determined by the Sheather-Jones method (Sheather and Jones (1991)) which proves to be the favorable choice in the comparison of Jones et al. (1996). This estimator is applied to the industry means of the first five years (1958-62) and the last five years (1992-96) of the logged value added shares. The averaging makes the estimates less sensitive with respect to shocks that are specific to a single year. Figure 1 shows these density estimates in the left panel by the dashed and solid lines, respectively.

Figure 1

Shape of the Density of the Value Added Shares
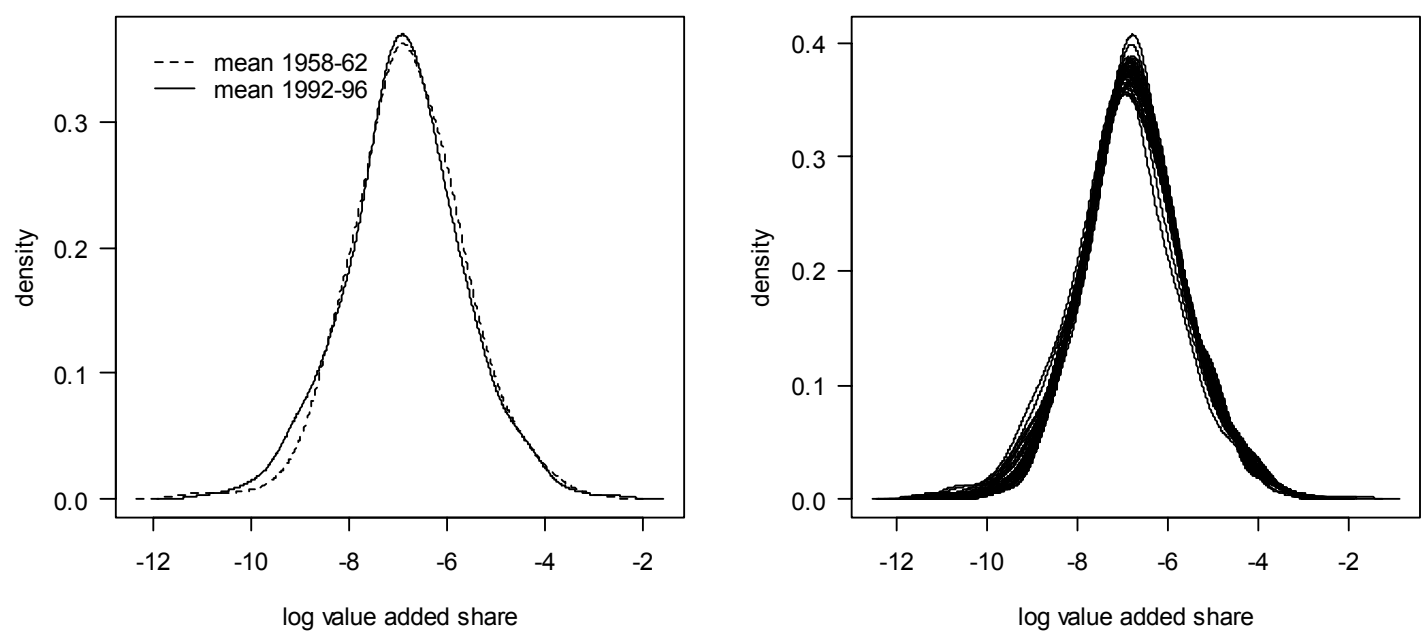

Ist is immediate that the shape of the density is approximately the same in the first and last five years which points to the stationarity of the stochastic process of the value added shares. However, there may be large changes of the shape of the distribution during the intervening years. To assure that this is not the case, the densities for all years are plotted simultaneously 
in the right panel of figure 1. This shows that the stability of the distribution is not the result of an accidental conformity of the distributions at the beginning and the end of the sample period but instead holds consistently during the whole sample period.

The stationarity of the shape of the distribution does not imply by itself a low intensity of structural change. Of course, stationarity may be consistent with nearly constant shares of the industries, but stationarity may also be associated with substantial intra-distributional changes that compensate each other so that the shape of the distribution is preserved. To get an impression of the intra-distribution dynamics we estimate the transition density nonparametrically as explained in Quah (1996a, p. 117). Applied to the value added share dynamics, figure 2 shows the respective transition density. Each slice through the plot along the $x$-axis represents the density of the log mean value added shares of the last five years conditional on a specific log mean value added share of the first five years on the $y$-axis.

Figure 2

Nonparametric Transition Density

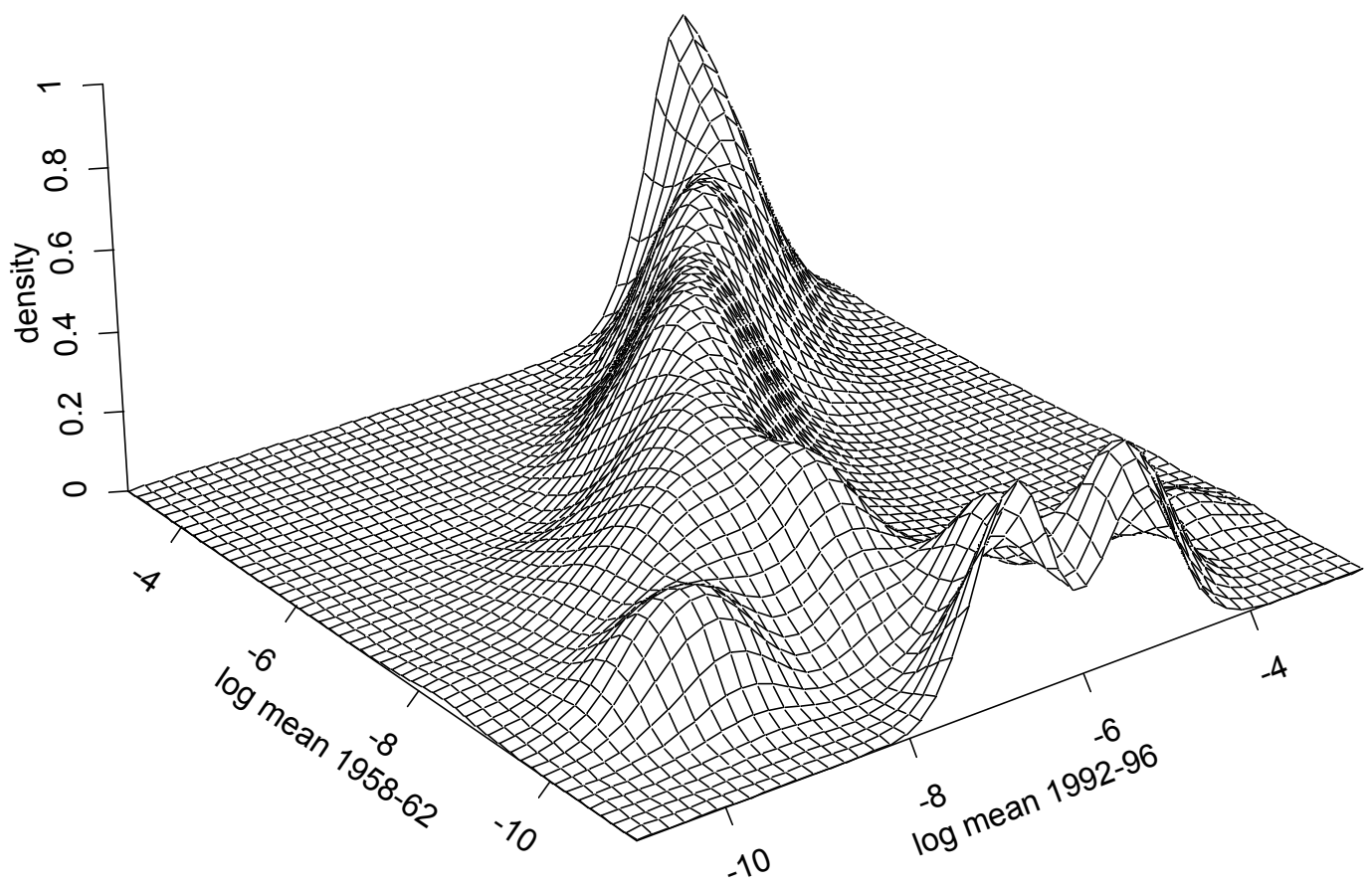


Clearly visible from the ridge along the diagonal in the figure is the general tendency of persistence of the value added shares. However, the dispersion of the distribution shows that intra-distributional changes and thus structural change are prevalent, especially in the range of relatively low value added shares. Particularly interesting in this respect is the shape of the density at the bottom of the figure in the region of log mean value added shares 1958-62 below the value -10 . The growth of the four industries that shape the density in this region was much faster than that of the rest of the manufacturing sector. Remarkably, the four industries consist of computer storage devices (SIC 3572), computer terminals (SIC 3575), computer peripheral equipment (SIC 3577) and magnetic and optical recording media (SIC 3695), all of which are strongly related to the computer revolution.

\section{Theoretical Model}

The statistical results reported in the preceding section show that the stochastic process that governs the real value added shares of the four-digit manufacturing industries is associated with a stationary distribution together with substantial intra-distributional changes. These intra-distributional changes are the trace that the process of structural change leaves in the data. The theoretical model constructed in this section to explain these empirical results is built around a Markov process that specifies a law of motion governing the dynamics of the value added shares. The transition law of this Markov process is assumed to depend on the productivity of the industries relative to a benchmark. Specifically, a probabilistic search mechanism generates the distribution of the productivity dynamics which is subsequently used to derive the transition density of the Markov process.

The dynamics of the the real value added shares are supposed to be governed by a general Markov process with a law of motion

$$
s_{t}=h\left(s_{t-1}, \beta\left(\Delta \tilde{a}_{t}\right)\right)
$$

where $h:[0,1] \times \mathbb{R} \rightarrow[0,1]$ is a continuous function that is increasing in both arguments and $t=1,2, \ldots$ indexes time proceeding in discrete steps. The function $\beta\left(\Delta \tilde{a}_{t}\right)$ depends on the 
change of the productivity of an industry relative to its competitors $\Delta \tilde{a}_{t} \in \mathbb{R}$ as an operationalization of its competitiveness. It thus controls the influence that the change of productivity relative to the competitors exerts on the change of the real value added shares. This law of motion specifies a sequence of random variables of a specific industry which is considered simply as a dummy argument. In the distribution dynamics framework the universe of all manufacturing industries and their heterogeneity is represented by the associated sequence of distributions which will be derived below.

Since shares are by definition bounded in the interval $[0,1]$ concrete functional specifications are usually quite restrictive since they are required to map $[0,1]$ into itself. The specification that is analyzed in the main body of this paper is based on the power function $s_{t}=s_{t-1}^{\beta\left(\Delta \tilde{a}_{t}\right)}$, where the function $\beta(\cdot)$ has the properties of being positive $\beta(x) \geq 0$ and decreasing in its argument $\beta^{\prime}(x) \leq 0$ for all $x \in \mathbb{R}$ with the prime denoting the first derivative. This ensures that a larger positive change of relative productivity leads to an increasing value added share. The limiting properties of the function are assumed to be $\lim _{x \rightarrow \infty} \beta(x)=0$ and $\lim _{x \rightarrow-\infty} \beta(x)=\infty$. An alternative specification based on the logistic function is briefly discussed in the appendix. That specification is, however, associated with a worse fit to the data.

Associated with this process is a law of motion for the probability measure $v$ of the value added shares

$$
v_{t}(S)=\int_{[0,1]} P(s, S) v_{t-1}(d s)
$$

where $P(s, S)=\operatorname{Pr}\left(s_{t} \in S \mid s_{t-1}=s\right)$ denotes the so-called transition kernel. This transition kernel is required to be a measurable function in the first argument and a probability measure in the second argument. It provides a complete probabilistic description of all possible transitions from any particular state $s \in[0,1]$ to any other part of the state space $S \subseteq[0,1]$.

The derivation of the transition kernel is based on a probabilistic search mechanism in which the number of independent ideas that are generated by the firms of a specific industry is assumed to depend on the value added share of this industry $\lambda(s)$ with $\lambda(s) \in\{1,2, \ldots\}$. A larger value added share allows the firms in the industry to devote more ressources to $R \& D$ 
activities and in addition enhances the possibilities for learning-by-doing driven productivity improvements. The relation of the value added share to the generated number of ideas is assumed to satisfy the conditions $\lambda(s) \geq 0, \lambda(0)=0$ and $\lambda^{\prime}(s) \geq 0$ as well as the Inada-type conditions $\lambda^{\prime}(0)=\infty$ and $\lambda^{\prime}(1)=0($ see Inada $(1963))$.

Each of these ideas is associated with a potential relative productivity improvement of $\Delta \tilde{a}_{t}$ which is drawn from a distribution with cumulative distribution function (cdf) $F\left(\Delta \tilde{a}_{t}\right)$. This distribution is called the search distribution in what follows. It could be made dependent on the past relative productivity level which would allow for the possibility of increasing or diminishing technological opportunities, but we resign from doing so for simplicity.

At the end of the period the idea that is associated with the largest relative productivity improvement (which must not necessarily be positive) is selected and implemented. The distribution of this largest relative productivity improvement can straightforwardly be established using the theory of order statistics (see e.g. Arnold et al. (1992)). Accordingly, the cdf $G$ of relative productivity growth associated with the implemented idea is

$$
G\left(\Delta \tilde{a}_{t}\right)=F\left(\Delta \tilde{a}_{t}\right)^{\lambda\left(s_{t-1}\right)}
$$

with density function

$$
g\left(\Delta \tilde{a}_{t}\right)=\lambda\left(s_{t-1}\right) F\left(\Delta \tilde{a}_{t}\right)^{\lambda\left(s_{t-1}\right)-1} \cdot f\left(\Delta \tilde{a}_{t}\right),
$$

where $f\left(\Delta \tilde{a}_{t}\right)$ is the density function associated with $F\left(\Delta \tilde{a}_{t}\right)$. Recall that the function $\lambda\left(s_{t-1}\right)$ represents the number of ideas generated, depending on the lagged value added share.

Now we are in the position to derive the transition kernel by combining the productivity distribution $G$ with the Markov process for the value added share dynamics $s_{t}=h\left(s_{t-1}, \beta\left(\Delta \tilde{a}_{t}\right)\right)$. Theorem 8.9 of Stokey and Lucas (1989) is the key result for this task; the same approach can be found in Loury (1981). The basic idea is to construct a set that represents all possible changes of relative productivity that are associated with the transition to a specific interval of shares $S=\left[s_{1}, s_{2}\right] \subseteq[0,1]$ starting from a specific share $s \in[0,1]$. In the present case such a set is given by $A=h^{-1}(s, S)=\{\Delta a \in \mathbb{R}: h(s, \beta(\Delta a)) \in S\}$. The probability of the set $A$ is equal 
to $\operatorname{Pr}\left(s_{t} \in S \mid s_{t-1}=s\right)=P(s, S)$ and can be calculated explicitly from the productivity distribution. Thus, the transition kernel can be derived by integrating the productivity distribution over the set $A, P(s, S)=\int_{A} d G\left(\Delta \tilde{a}_{t}\right)$.

The set $A$ is in the case of the power specification given by

$$
A=\left[b\left(\frac{\ln s_{1}}{\ln s}\right), b\left(\frac{\ln s_{2}}{\ln s}\right)\right]
$$

where $b(\cdot)$ denotes the inverse function of $\beta(\cdot)$ and is decreasing in its argument since this property has been assumed above for the function $\beta(\cdot)$. The resulting transition kernel is

$$
P\left(s,\left[s_{1}, s_{2}\right]\right)=G\left(b\left(\frac{\ln s_{2}}{\ln s}\right)\right)-G\left(b\left(\frac{\ln s_{1}}{\ln s}\right)\right)=F\left(b\left(\frac{\ln s_{2}}{\ln s}\right)\right)^{\lambda(s)}-F\left(b\left(\frac{\ln s_{1}}{\ln s}\right)\right)^{\lambda(s)} .
$$

The empirical exploration of the value added shares above has shown that these seem to be associated with a stationary distribution. Therefore the transition kernel should be associated with a stationary Markov process for the value added shares. Such a process can be mathematically characterized by a stationary (or invariant) measure $v^{*}$ with the property $v^{*}(S)=\int_{[0,1]} P(s, S) v^{*}(d s)$. Another important notion in this respect in the concept of ergodicity, meaning the convergence of a stochastic process to the stationary (or invariant) distribution, irrespective of the initial distribution. These properties can be established by checking the conditions of Hopenhayn and Prescott (1992) which are quite easy to verify directly from the properties of the transition kernel and thus ideally suited to the case at hand.

To establish the existence of a stationary distribution, corollary 4 of Hopenhayn and Prescott (1992) requires that the state-space is a compact metric space with a minimum element which is evidently the case for the interval $[0,1]$. In addition, the transition distribution has to be increasing in the sense of first-order stochastic dominance (see Wolfstätter (1999, ch. 4)). For the power specification the cdf associated with the transtition kernel is

$$
P\left(s,\left[0, s_{2}\right]\right)=F\left(b\left(\frac{\ln s_{2}}{\ln s}\right)\right)^{\lambda(s)}
$$

since $\lim _{s_{1} \backslash 0} \ln s_{1} / \ln s=\infty$ and $\lim _{x \rightarrow \infty} b(x)=-\infty$ by the properties of $\beta(\cdot)$. 
The transition kernel is increasing in the above defined sense of first-order stochastic dominance since for $s \geq s^{\prime}$ it is true that $b\left(\frac{\ln s_{2}}{\ln s}\right) \leq b\left(\frac{\ln s_{2}}{\ln s^{\prime}}\right)$ because of $b^{\prime}(\cdot) \leq 0$ and therefore $F\left(b\left(\frac{\ln s_{2}}{\ln s}\right)\right) \leq F\left(b\left(\frac{\ln s_{2}}{\ln s^{\prime}}\right)\right)$ since a cdf is monotonically increasing and because of $\lambda(s) \geq \lambda\left(s^{\prime}\right)$ we finally obtain $F\left(b\left(\frac{\ln s_{2}}{\ln s}\right)\right)^{\lambda(s)} \leq F\left(b\left(\frac{\ln s_{2}}{\ln s^{\prime}}\right)\right)^{\lambda\left(s^{\prime}\right)}$.

For the uniqueness and stability and thus ergodicity of the transition kernel theorem 2 of Hopenhayn and Prescott (1992) claims that the state-space has to contain both a lower and an upper bound which is trivially satisfied for $[0,1]$ and that the transition kernel has to be increasing which has been just verified in the preceding paragraph. Moreover, a so-called monotone mixing condition (MMC) has to be satisfied which essentially requires that each part of the state-space can be reached from any starting point after a certain number of periods. The MMC condition thus essentially requires a considerable amount of mobility below the stationary distribution. However, this condition is not satisfied for the power specification because $s=0$ and $s=1$ are absorbing states and thus

$$
P\left(1,\left[0, s^{*}\right]\right)=\lim _{s \nearrow 1} P\left(s,\left[0, s^{*}\right]\right)=\lim _{s \nearrow 1} F\left(b\left(\frac{\ln s^{*}}{\ln s}\right)\right)^{\lambda(s)}=F(b(\infty))^{\lambda(1)}=0 \forall s^{*} \in[0,1]
$$

and

$$
P\left(0,\left[s^{*}, 1\right]\right)=\lim _{s \searrow 0} P\left(s,\left[s^{*}, 1\right]\right)=\lim _{s \searrow 0} F\left(b\left(\frac{\ln 1}{\ln s}\right)\right)^{\lambda(s)}-F\left(b\left(\frac{\ln s^{*}}{\ln s}\right)\right)^{\lambda(s)}=0 \forall s^{*} \in[0,1]
$$

by the properties of the inverse function $b(\cdot)$ and the limiting behavior of the logarithm. These results show that in the case of the power specification a stationary distribution exits but is neither unique nor stable on the state-space $[0,1]$. The reason is that the states 0 and 1 are absorbing states and will never be left once they are reached by the process. On the statespace $(0,1)$, however, these absorbing states are excluded and it is intuitively clear that the Markov process induced by the power specification is irreducible (all parts of the state-space can be reached from any starting point with positive probability), recurrent (all parts of the state-space are guaranteed to be reached from every starting point in a finite number of steps) and aperiodic (there are no cyclical movements between any parts of the state-space). According to these more abstract concepts, the results outlined in Meyn and Tweedie (1993) and Tweedie (2001) imply that the Markov process has a stationary distribution and is ergodic. Thus, in this case a unique stationary distribution exists and the process converges to this 
distribution from any arbitrary initial distribution. In our sample the upper bound of unity for the value added share will never be reached and the asbestos industry (SIC 3292), which is the single case that attains the lower bound of zero, has been eliminated from the data.

It is worth emphasizing that the above results are derived with only minimal assumptions about the properties of the functions $\beta(\cdot)$ and $\lambda(\cdot)$ and the search distribution $F$. This ensures that the results are robust for wide classes of functions and distributions. In the appendix another specification based on the logistic function is analyzed which is slightly more favorable from a theoretical point of view since the ergodicity of the transition kernel can be established even on the state-space $[0,1]$. Interestingly, as the following sections will demonstrate, the power specification fits much better to the data, however.

\section{Estimation of the Transition Density}

For the desired maximum likelihood estimation of the transition density it is unfortunate from a numerical point of view that the number of ideas is an integer magnitude and not a continuous variable. This deficiency can be remedied in a very appealing way by treating the number of ideas itself as a random draw from a Poisson distribution with expectation $\lambda\left(s_{t-1}\right) \in \mathbb{R}_{+}$and deriving the cdf of the distribution of productivity growth by the construction of a mixture distribution. To get a valid distribution for the number of ideas with support starting at unity, the Poisson distribution has to be truncated from below at unity.

From the Poisson distribution with density $\operatorname{Pr}(J=j)=\lambda^{j} \exp (-\lambda) / j !, j=0,1,2, \ldots$ we know that $\operatorname{Pr}(J=0)=\exp (-\lambda)$ and hence that $\operatorname{Pr}(J \geq 1)=1-\exp (-\lambda)$. Therefore the truncated Poisson distribution has the density function $\operatorname{Pr}(J=j \mid J \geq 1)=\lambda^{j} \exp (-\lambda) /(j ! \cdot(1-\exp (-\lambda)))$ on the support $j=1,2, \ldots$.

The mixture distribution is formed by a weighted average of the distribution of the largest order statistic with the truncated Poisson probabilities as weighting factors. The cdf of this mixture distribution is then 


$$
\begin{aligned}
G\left(\Delta \tilde{a}_{t}\right) & =\sum_{j=1}^{\infty} \frac{\lambda\left(s_{t-1}\right)^{j} \exp \left(-\lambda\left(s_{t-1}\right)\right) / j !}{1-\exp \left(-\lambda\left(s_{t-1}\right)\right)} \cdot F\left(\Delta \tilde{a}_{t}\right)^{j} \\
& =\frac{\exp \left(-\lambda\left(s_{t-1}\right)\right)}{1-\exp \left(-\lambda\left(s_{t-1}\right)\right)} \cdot \sum_{j=1}^{\infty} \frac{\left(\lambda\left(s_{t-1}\right) F\left(\Delta \tilde{a}_{t}\right)\right)^{j}}{j !} \\
& =\frac{\exp \left(-\lambda\left(s_{t-1}\right)\right)}{1-\exp \left(-\lambda\left(s_{t-1}\right)\right)} \cdot\left(\exp \left(\lambda\left(s_{t-1}\right) F\left(\Delta \tilde{a}_{t}\right)\right)-1\right) \\
& =\frac{\exp \left(\lambda\left(s_{t-1}\right) F\left(\Delta \tilde{a}_{t}\right)\right)-1}{\exp \left(\lambda\left(s_{t-1}\right)\right)-1},
\end{aligned}
$$

where the result $\sum_{j=1}^{\infty} \frac{x^{j}}{j !}=\sum_{j=0}^{\infty} \frac{x^{j}}{j !}-1=\exp (x)-1$ has been used in the second to last line.

This truncated Poisson mixture distribution is associated with the density function

$$
g\left(\Delta \tilde{a}_{t}\right)=\frac{\exp \left(\lambda\left(s_{t-1}\right) F\left(\Delta \tilde{a}_{t}\right)\right)}{\exp \left(\lambda\left(s_{t-1}\right)\right)-1} \cdot \lambda\left(s_{t-1}\right) f\left(\Delta \tilde{a}_{t}\right)
$$

and leads to the transition distribution

$$
P\left(s,\left[0, s_{2}\right]\right)=\frac{\exp \left(\lambda(s) F\left(b\left(\frac{\ln s_{2}}{\ln s}\right)\right)\right)-1}{\exp (\lambda(s))-1}
$$

The main advantage of the truncated Poisson mixture distribution is that it avoids problems with the integer nature of the number of ideas generated. From a numerical point of view, imposing this integer restricition would make the subsequent maximization of the likelihood function much more complicated. Moreover, the cdf of the mixture distribution has shape characteristics that are very similar to those of the productivity distribution derived from the distribution of the largest oder statistics for a wide range of different parameter values. For these reasons, we favor the truncated Poisson mixture distribution as the basis for deriving the transition kernel on which the maximum likelihood estimation of the parameters is based.

Figure 3 compates the cumulative distribution functions of the largest order statistic (solid line) and the truncated Poisson mixture (dotted line) where the search distribution $F$ is assumed to be normal with mean $\alpha$ and unit standard deviation for various values of $\alpha$ and $\lambda$. Both distributions agree very closely and this finding also holds for a wide variety of other parameter values and modified assumptions for the search distribution. As general tendency 
we observe that the mean of both distributions is higher for larger values of $a$ and that the discrepancy between the two distributions (especially at the lower tail) rapidly vanishes as $\lambda$ grows.

Figure 3

Comparison of Cumulative Distribution Functions
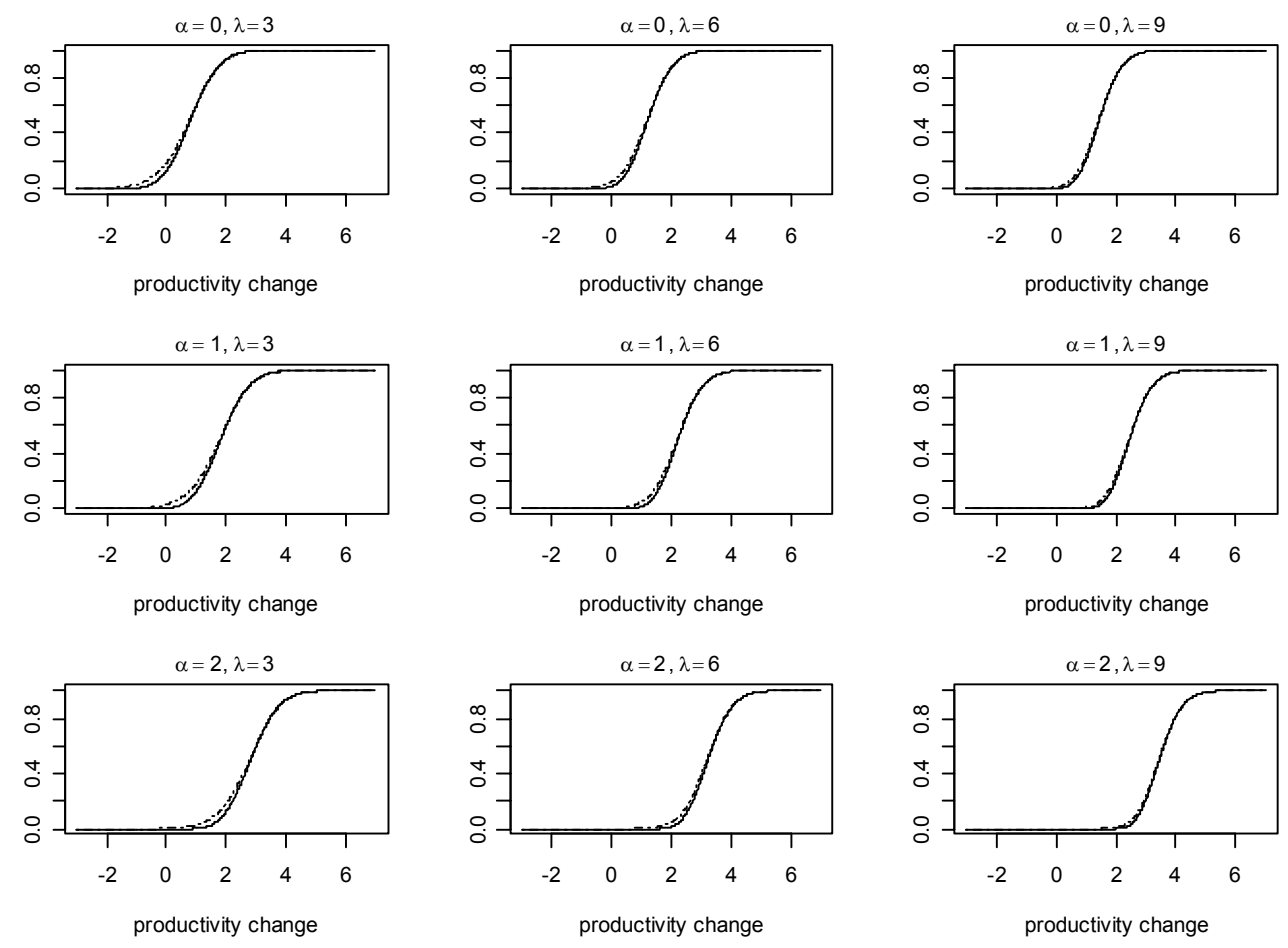

The transition distribution derived by the mixing procedure now serves as the basis for the estimation of the transition density by maximum likelihood. The loglikelihood function is

$$
\ln L=\sum_{i=1}^{N} \lambda\left(s_{t-1}\right) F\left(\Delta \tilde{a}_{t}\right)-\ln \left(\exp \left(\lambda\left(s_{t-1}\right)\right)-1\right)+\ln \lambda\left(s_{t-1}\right)+\ln f\left(\Delta \tilde{a}_{t}\right)
$$

The implementation requires that certain elements of the loglikelihood function need to be specified more concretely. For the search distribution a normal distribution with mean $\alpha_{0}$ and variance $\alpha_{1}$ is chosen, leading to $F\left(\Delta \tilde{a}_{t}\right)=\Phi\left(\left(\Delta \tilde{a}_{t}-\alpha_{0}\right) / \alpha_{1}\right)$, where $\Phi(\cdot)$ denotes the standard normal cdf. The parameter of the (truncated) poisson mixture weights is supposed to depend linearly on the lagged logged value added share, $\lambda\left(s_{t-1}\right)=\lambda_{0}+\lambda_{1} \ln s_{t-1}$. 
In the case of the power specification we assume $\beta\left(\Delta \tilde{a}_{t}\right)=\beta_{0}-\beta_{1} \Delta \tilde{a}_{t}$ with the corresponding inverse function $b\left(\ln s_{t} / \ln s_{t-1}\right)=\beta_{1}^{-1}\left(\beta_{0}-\ln s_{t} / \ln s_{t-1}\right) .{ }^{3}$ Taking all this together the transition distribution is

$$
P\left(s_{t-1},\left[0, s_{t}\right]\right)=\frac{\exp \left(\left(\lambda_{0}+\lambda_{1} \ln s_{t-1}\right) \Phi\left(\left(\beta_{0}-\ln s_{t} / \ln s_{t-1}\right) /\left(\alpha_{1} \beta_{1}\right)-\alpha_{0} / \alpha_{1}\right)\right)-1}{\exp \left(\lambda_{0}+\lambda_{1} \ln s_{t-1}\right)-1}
$$

and the conditional density of the logged value added shares is

$$
\begin{aligned}
p\left(\ln s_{t} \mid \ln s_{t-1}\right)= & \frac{\exp \left(\left(\lambda_{0}+\lambda_{1} \ln s_{t-1}\right) \Phi\left(\left(\beta_{0}-\ln s_{t} / \ln s_{t-1}\right) /\left(\alpha_{1} \beta_{1}\right)-\alpha_{0} / \alpha_{1}\right)\right)}{\exp \left(\lambda_{0}+\lambda_{1} \ln s_{t-1}\right)-1} \\
& \cdot\left(\lambda_{0}+\lambda_{1} \ln s_{t-1}\right) \phi\left(\left(\beta_{0}-\ln s_{t} / \ln s_{t-1}\right) /\left(\alpha_{1} \beta_{1}\right)-\alpha_{0} / \alpha_{1}\right) \\
& \cdot\left(-1 /\left(\alpha_{1} \beta_{1} \ln s_{t-1}\right)\right) .
\end{aligned}
$$

The last (Jacobian) factor in the transition density is guaranteed to be nonnegative since $s_{t-1}$ is bounded between zero and unity. The transtition density forms the basis for the loglikehood function of all industries $i=1, \ldots, N$

$$
\ln L=\sum_{i=1}^{N} \ln p\left(\ln s_{i t} \mid \ln s_{i t-1}\right)
$$

where $s_{i t}$ and $s_{i t-1}$ denote the averages of the real value added shares of the first and the last five years of data available used in the empirical implementation. This loglikelihood function is maximized with respect to the six parameters $a_{0}, \alpha_{1}, \beta_{0}, \beta_{1}, \lambda_{0}$ and $\lambda_{1}$. Numerically the maximization is performed using the BFGS method of Broyden, Fletcher, Goldfarb and Shanno (see e.g. Dennis and Schnabel (1983, ch. 9) for an exposition) which as a quasiNewton method seems to be best suited to the smooth loglikelihood function at hand. A large number of randomly chosen starting values have been tried in order to find the global maximum of the loglikelihood function. Other methods, like conjugate gradient and the Nelder-Mead simplex, proved here to be less satisfactory compared to the BFGS method.

Unfortunately this estimation problems suffers from a parameter identification problem so that it makes no sense to report the individual parameter estimates. This notwithstanding, the

\footnotetext{
3 Obviously, this specification does not match the limiting properties postulated above. However, this deficiency is accepted here since this specification is very convenient in the subsequent application of quantile regression.
} 
overall fit of the resulting estimate of the transition density is remarkable. Plugging the parameter estimates into the formula for $\ln p\left(\ln s_{t} \mid \ln s_{t-1}\right)$ and evaluating this function for the relevant range of value added shares gives the plot of the fitted transition density that is depicted in the following figure 4.

Figure 4

Transition Density for Power Specification (ML Fit)

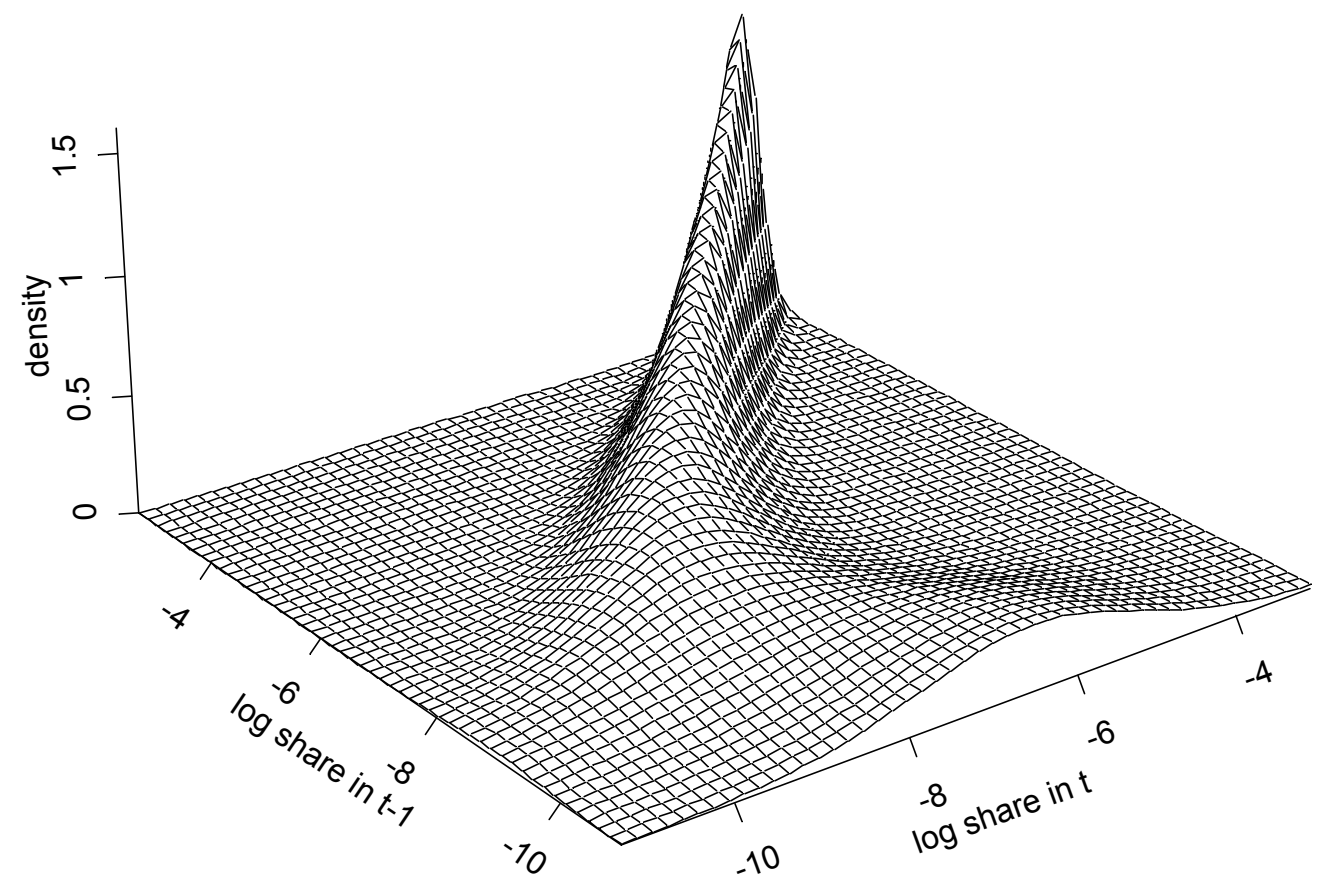

Comparing this plot with the nonparametric estimate of the transition density in figure 2 above reveals the striking similarity of both densities. Both densities are characterized by a dominant ridge along the main diagonal which increases for larger value added shares. Thus the theoretical model encompasses the tendency towards a relatively higher degree of persistence in the case of industries with comparably larger value added shares. The theoretical model is yet flexible enough to capture the imprint of the computer revolution discussed above. Like in the case of the nonparametric estimate there appears an exceptional shape of the density function at the bottom of the figure for log value added shares below -10 in $t-1$. 
Since the parameter identification problem effectively precludes the test of the relation between productivity change and the value added share dynamics this issue will be investigated in the remainder of this paper using the approach of quantile regression. Before we turn to this, we briefly describe the method used to compute the measure of relative productivity already used in the theory from real data.

\section{Productivity Measurement}

Productivity change is computed here by the Malmquist index of total factor productivity together with data envelopment analysis. This nonparametric approach quantifies productivity change by computing radial distances relative to piece-wise linear frontier production functions that are formed by the most productive industries of the sample. Thereby the approach accounts for both efficiency change below this frontier function and shifts of the frontier function itself. Compared to the Solow residuals (Solow (1957)) usually employed in the literature, this procedure has several notable advantages. The main advantage is that no behavioral assumptions such as profit maximization and no presumption of competitive markets are required. No price data are required for the calculation of the Malmquist index, instead it relies exclusively on quantity data. The nonparametric nature makes the approach also less restrictive than the index number or regression methods used in the literature and it is therefore expected to achieve a better fit to the data. The approach is described in detail in the technical literature on productivity measurement (see e.g. Färe et al. (1998)) as well as in the significant economic applications by Färe et al. (1994) and Kumar and Russell (2002) among many others.

For a brief formal description of the approach, let $\boldsymbol{y}_{i j}$ and $\boldsymbol{x}_{i j}$ denote vectors that comprise the output and input quantities used by industry $i$ in period $j$, respectively. ${ }^{4}$ Further, let

$$
S(j)=\left\{\left(\boldsymbol{x}_{i j}, \boldsymbol{y}_{i j}\right): \boldsymbol{x}_{i j}>\mathbf{0} \text { can produce } \boldsymbol{y}_{i j}>\mathbf{0}, \forall i=1, \ldots, N\right\}
$$

\footnotetext{
${ }^{4}$ Here $j$ is used to index the single years between 1958 and 1996. This should not be confused with the notation $t$ and $t+1$ which refers to the industry averages over the first and last five years of the sample, respectively.
} 
denote the technology set representing the production possibilities available in the manufacturing sector in period $j$ which is spanned by the $N$ industries. Based on this a radial distance function

$$
D_{i p}\left(\boldsymbol{x}_{i q}, \boldsymbol{y}_{i q}\right)=\left(\sup \left\{\phi:\left(\boldsymbol{x}_{i q}, \phi \boldsymbol{y}_{i q}\right) \in S(p)\right\}\right)^{-1}
$$

is defined as the reciprocal of the maximum augmentation of the output values in period $q$ (holding inputs constant) that is required to reach a boundary point of the technology set in period $p$. Setting $p$ and $q$ alternately to $j$ and $j+1$ four different distance functions can be computed. From these the Malmquist index of productivity change can be assembled as

$$
M_{i, j+1}\left(\boldsymbol{x}_{i j}, \boldsymbol{y}_{i j}, \boldsymbol{x}_{i, j+1}, \boldsymbol{y}_{i, j+1}\right)=\left[\frac{D_{i j}\left(\boldsymbol{x}_{i, j+1}, \boldsymbol{y}_{i, j+1}\right)}{D_{i j}\left(\boldsymbol{x}_{i j}, \boldsymbol{y}_{i j}\right)} \frac{D_{i, j+1}\left(\boldsymbol{x}_{i, j+1}, \boldsymbol{y}_{i, j+1}\right)}{D_{i, j+1}\left(\boldsymbol{x}_{i j}, \boldsymbol{y}_{i j}\right)}\right]^{1 / 2} .
$$

The first fraction in the square brackets is formed by the ratio of the distances of the observation of industry $i$ in period $j$ and $j+1$, respectively, to the frontier of the technology set in period $j$. Given that industry $i$ is closer to the frontier function of period $j$ in period $j+1$ than it is in period $j$, this ratio is larger than unity, implying positive productivity change. The interpretation of the second fraction is analogous with respect to the frontier function of period $j+1$. Since there is no special reason to choose the frontier function of period $j$ over that of period $j+1$ as the benchmark for the measurement of productivity change, the Malmquist index is defined as the geometric average of both. Constructed in this way, the Malmquist index indicates positive (negative) growth of total factor productivity between periods $j$ and $j+1$ if $M_{i, j+1}$ is larger (smaller) than unity.

With real data the distance functions are calculated by data envelopment analysis (see Charnes et al. (1994)) as the solution of the linear programming problems in curly brackets

$$
D_{i p}\left(\boldsymbol{x}_{i q}, \boldsymbol{y}_{i q}\right)=\left[\max \left\{\phi: \phi \cdot \boldsymbol{y}_{i q} \leq \sum_{h=1}^{N} \lambda_{h} \boldsymbol{y}_{h p} ; \sum_{h=1}^{N} \lambda_{h} \boldsymbol{x}_{h p} \leq \boldsymbol{x}_{i q} ; \lambda_{1}, \ldots, \lambda_{N} \geq 0\right\}\right]^{-1}
$$

which does not invoke any assumptions about the functional form of the production relationship and by this nonparametric feature gives the whole procedure a great amount of flexibility. 
Returns to scale are assumed to be constant which seems to be reasonably appropriate for U.S. manufacturing industries according to Basu and Fernald (1997). ${ }^{5}$

The data used to calculate the Malmquist index are again taken from the NBER-CES manufacturing industry database. The nonparametric productivity measurement is performed with the following specification of the output variable and the six labor, capital and material/energy input variables (the abbreviations in square brackets refer again to those defined in the data appendix of Bartelsman and Gray (1996)). Output is represented by the real value of shipments [VSHIP/PISHIP], labor inputs are the number of non-production workers [EMP-PRODE] and production worker hours [PRODH] and capital inputs are the real capital stocks for equipment [EQUIP] and structures [PLANT], respectively. Energy input is measured by the real expenditures on fuels and electricity [ENERGY/PIEN] and material input is represented by the real cost of materials other than electricity and fuels [MATCOST/PIMAT-ENERGY/PIEN].

The calculations of the Malmquist index for each year separately result in a balanced panel of total factor productivity changes $M_{i j}(i=1, \ldots, N, j=2, \ldots, T)$ larger than zero for each of the $N$ industries and $T=39$ years spanning the period 1958-96. The specific measure of productivity change of the industries relative to their competitors used subsequently is the geometric mean over the whole sample period identical to the average annual growth rate of total factor productivity, computed as $\Delta \tilde{a}_{i t}=\left(\prod_{j=2}^{T} M_{i j}\right)^{1 /(T-1)}-1$ for each industry $i \in\{1, \ldots, N\}$. Although inessential at this place, the time index $t$ is retained to indicate that this measure of productivity change is the average over the whole sample period consistent with the variable used in the theoretical part.

In his recent survey Balk (2003) shows that the total factor productivity change is related to both profitability and price changes and in particular that total factor productivity change measures the real component of profitability change. Thus the change of total factor productivity as calculated here can be alternatively interpreted as an encompassing measure of the

\footnotetext{
5 This procedure is entirely deterministic. There exists an alternative econometric approach to the estimation of fontier functions that promises to be able to divide measurement error from the productivity measure (see e.g. Greene 1993). However, the Monte Carlo studies of Banker et al. (1993) and Ruggiero (1999) show that this advantage of the econometric appoach over DEA is present only in very large samples, whereas productivity and measurement error frequently get confused in small to medium sized samples.
} 
change of the competitiveness of industries. See also Lipsey and Carlaw (2000) for additional discussion of the meaning of total factor productivity change.

\section{Quantile Regression Estimates}

Quantile regression ${ }^{6}$ introduced by Koenker and Bassett (1978) is a powerful tool for the characterization of the entire distribution of a dependent variable given a set of regressors and not just its mean as in the case of least squares regression. Therefore, quantile regression has the potential to uncover differences in the response of the dependent variable to changes of the regressors at different points of the conditional distribution of the dependent variable and by that provides a large amount of information about the heterogeneity of the oberservations. Moreover, coefficient estimates obtained with quantile regression are more robust with respect to outliers of the dependent variable and in the case of nonnormal errors quantile regression estimates may be more efficient than least squares estimates (see Buchinsky (1998, p. 89) and Fitzenberger et al. (2001, p. 1)).

In contrast to the case of ordinary linear regression that solves the least squares problem $\min _{\boldsymbol{\beta} \in \mathbb{R}^{k}} \sum_{i=1}^{N}\left(y_{i}-\boldsymbol{x}_{i}^{\prime} \boldsymbol{\beta}\right)^{2}$, where $\boldsymbol{x}_{i}$ is the $k$-vector of the regressors of observation $i=1, \ldots, N$, and estimates the conditional mean of $y$ given $\boldsymbol{x}$, ordinary quantile regression solves the problem $\min _{\boldsymbol{\beta} \in \mathbb{R}^{k}} \sum_{i=1}^{N} \rho_{\tau}\left(y_{i}-\boldsymbol{x}_{i}^{\prime} \boldsymbol{\beta}\right)$, where the sum of asymmetrically weighted absolute residuals is minimized. The weighting of the residuals is controlled by the so-called "check function" $\rho_{\tau}(u)=u \cdot(\tau-I(u<0))$ where $I(\cdot)$ denotes the usual indicator function which is equal to unity if $u<0$ and zero otherwise.

Quantile regressions can also be estimated in the context of a generalized instrumental variables estimator (GIVE). This builds upon the work of Chen and Portnoy (1996). ${ }^{7}$ In this procedure the endogenous regressors are first projected on to the space spanned by the exogenous regressors and the instruments, which are uncorrelated with the error terms by assumption. Defining $\boldsymbol{X}$ as the $N \times k$-matrix of all (endogous and exogenous) regressors and $\boldsymbol{W}$ as the

\footnotetext{
${ }^{6}$ This brief exposition of the basics of quantile regression in this section draws from the very useful survey articles of Buchinsky (1998), Koenker (2000) and Koenker and Hallock (2001).

7 See also Arias et al. (2001). I am indebted to Omar Arias for kindly providing his S rountines for the instrumental variables estimation of quantile regression.
} 
$N \times l$-matrix (with $l>k$ ) of both exogenous regressors and instruments, this projection is equivalent to the matrix operation $\hat{\boldsymbol{X}}=\boldsymbol{W}\left(\boldsymbol{W}^{\prime} \boldsymbol{W}\right)^{-1} \boldsymbol{W}^{\prime} \boldsymbol{X}$ which does not affect the columns of the exogneous regressors but expresses the endogenous regressors as optimal (in the least squares sense) linear combinations of the variables in $\boldsymbol{W}{ }^{8}$ Since all variables in $\boldsymbol{W}$ are exogenous, the variables in the matrix $\hat{\boldsymbol{X}}$ are exogenous as well by the properties of linear projections (see Davidson and MacKinnon (2003, pp. 57ff.) for more on the geometry of linear projections). The matrix $\hat{\boldsymbol{X}}$ is subsequently used instead of the original regressors $\boldsymbol{X}$ in the estimation of the quantile regressions.

Among the variables we consider the productivity growth variable is suspect of endogeneity because it may be the case that not only productivity growth exerts an effect on the share dynamics but also that the share dynamics simultaneously influence productivity growth. Then the estimates of the ordinary regression quantiles are inconsistent in general. The other regressors are not affected by this endogeneity problem since they refer to the period $t-1$ (denoting again the industry mean of the first five years) and can thus be savely taken as predetermined. In the instrumental variable quantile regressions the variable $\Delta \tilde{a}_{i t}$ is instrumented by the lagged logged relative productivity level $\ln \tilde{a}_{i t-1}$ and its square $\left(\ln \tilde{a}_{i t-1}\right)^{2}$ in addition to the other predetermined variables. All instruments together are able to explain about 64 percent of the variation in $\Delta \tilde{a}_{i t}$ and can thus be considered not only as valid but also as relevant. The lagged productivity levels are computed as the industry averages of the first five years of a productivity measure that is computed by the Andersen and Petersen (1993) variant of data envelopment analysis using the same specification of the inputs and outputs as above (see Krüger (2005) for the details of the implementation of this method).

The covariance matrix of these regression quantiles is not valid since the regressors used are generated by the projection in the first step, however. Here, design-matrix bootstrapping of the whole two-step procedure is an appropriate way to compute valid confidence intervals for the regression quantiles estimated by instrumental variables. This approach usually performs well even if some forms of heteroskedasticity are present (see Buchinsky (1998), Hahn (1995) and Koenker (1994) for asymptotic as well as simulation results). The design-matrix bootstrap is based on randomly drawn samples (with replacement) of size $N$ from the original data

\footnotetext{
${ }^{8}$ Note that here the prime denotes matrix transposition.
} 
$\left(y_{i}, \boldsymbol{x}_{i}\right), i=1, \ldots, N$, denoted by $\left(y_{i}^{*}, \boldsymbol{x}_{i}^{*}\right), i=1, \ldots, N$. In the case of the instrumental variable quantile regression the bootstrap samples are drawn from $\left(y_{i}, \boldsymbol{x}_{i}, \boldsymbol{w}_{i}\right), i=1, \ldots, N$, where $\boldsymbol{w}_{i}$ denotes the $l$-vector of instrumental variables of the $i$ th observation. For each of these $B$ draws either the ordinary or the instrumental variable quantile regression is computed, resulting in $B$ different bootstrap estimates for $\hat{\beta}_{\tau b}^{*}=\left(\hat{\beta}_{1 \tau b}^{*}, \ldots, \hat{\beta}_{k \tau b}^{*}\right)^{\prime}, b=1, \ldots, B$. From these bootstrap estimates confidence intervals for each coefficient $j=1, \ldots, k$ and quantile $\tau$ can be established such that $\operatorname{Pr}\left(\beta_{j \tau} \in\left[L_{j \tau}^{*}, U_{j \tau}^{*}\right]\right) \approx 1-\alpha$, where the confidence limits $L_{j \tau}^{*}$ and $U_{j \tau}^{*}$ are simply the $\alpha / 2$ and $1-\alpha / 2$ quantiles of the respective bootstrap estimates $\left(\hat{\beta}_{j \tau 1}^{*}, \ldots, \hat{\beta}_{j \tau B}^{*}\right)$. All bootstrap estimates reported in this work are based on $B=10000$ replications.

Also available for regression quantiles is a goodness-of-fit statistic, proposed by Koenker and Machado (1999), which is a natural analog to $R^{2}$ in a least squares context and can be calculated for the $\tau$-th regression quantile by $R_{\tau}=1-\hat{V}_{\tau} / \tilde{V}_{\tau}$. Here, $\hat{V}_{\tau}=\min _{\boldsymbol{\beta} \in \mathbb{R}^{k}} \sum_{i=1}^{N} \rho_{\tau}\left(y_{i}-\boldsymbol{x}_{i}^{\prime} \boldsymbol{\beta}\right)$ is the minimized value of the unconstrained objective function for the $\tau$-th regression quantile and $\tilde{V}_{\tau}=\min _{\beta_{1} \in \mathbb{R}} \sum_{i=1}^{N} \rho_{\tau}\left(y_{i}-\beta_{1}\right)$ is the minimized value of the constrained objective function for the $\tau$-th regression quantile with only the intercept included as regressor. It is immediately clear that $R_{\tau}$ is bounded in $[0,1]$ for every $\tau$.

When stated in logs of the value added shares the power specification leads to the estimation equation

$$
\ln s_{i t}=\beta\left(\Delta \tilde{a}_{i t}\right) \ln s_{i t-1}+u_{i t}=\beta_{0} \ln s_{i t-1}-\beta_{1} \Delta \tilde{a}_{i t} \ln s_{i t-1}+u_{i t},
$$

where here $\beta\left(\Delta \tilde{a}_{i t}\right)=\beta_{0}-\beta_{1} \Delta \tilde{a}_{i t}$. In this specification productivity change appears interacted with the initial value added share, thereby ensuring that the shares are bounded within $[0,1]$. The results of the quantile regression estimates are summarized in the following figures for the ordinary quantile regression estimation and the quantile regression estimation involving instrumental variables. Each figure is divided in a number of separate graphs containing the coefficients estimates for the rountinely added intercept, the parameters $\beta_{0}, \beta_{1}$ and the goodness-of-fit measure $R$, reading from left to the right. The title of the first column denotes the dependent variable and the notations of the regressions are denoted at the ordinate of each separate graph. Therein the solid line originates from the connection of the coefficient 
estimates for the $\tau$-th quantile, where here $\tau \in\{0.05,0.1, \ldots, 0.95\}$ are considered as quantiles. This solid line is surrounded by two dotted lines indicating the upper and lower 95 percent confidence bounds. These confidence intervals are calculated by the design-matrix bootstrap based on $B=10000$ replications as outlined above. In addition, the horizontal dashed line represents the position of the least squares coefficient estimate (either OLS or GIVE). The last graph shows the goodness-of-fit statistic $R_{\tau}$ for each quantile. ${ }^{9}$

Turning to the power specification in figure 5 we observe that the intercept is negative and quite precisely estimated. The regression quantiles for the initial log value added shares are significantly positive throughout and show a declining shape. This implies that persistence is higher for lower quantiles compared to higher quantiles. In industries with relatively low actual value added shares, the quantile regression estimates tend towards unity which corresponds to the unit root case in the time series literature. The relevant null hypothesis for the unit root case is $H_{0}: \beta_{0 \tau}=1$. Based on the confidence intervals of the regression quantiles this null can not be rejected for industries with small value added shares in favor of the alternative $H_{1}: \beta_{0 \tau}<1$, whereas it can be rejected for industries with large value added shares. Thus, value added share dynamics of industries with large value added shares are in a sense "more stationary" compared to industries with small value added shares. ${ }^{10}$ This reasoning could also be interpreted in the spirit of a modified version of Gibrat's law applied to the value added shares. This law would hold if the estimates for $\beta_{0 \tau}$ are equal to unity consistent across all quantiles, implying that in this case growth is independent of size (with both growth and size measured in terms of value added shares). Since the estimates of $\beta_{0 \tau}$ are significantly below unity for the majority of quantiles, the modified version of Gibrat's law can be rejected for the manufacturing industries.

The regression quantiles for productivity growth intercated with the initial value added shares are positive and statistically significant across all quantiles. This implies that industries with higher productivity growth tend to reach a relatively larger value added share for a given initial value added share. The results give strong support for the positive relation between productivity growth and value added share dynamics asserted by the theoretical model,

\footnotetext{
9 This very efficient way to report the results of quantile regressions is due to Koenker and Hallock (2001).

${ }^{10}$ A similar discussion of the quantile estimates of interest rate dynamics is contained in section 5 of Koenker and Xiao (2004), although in that paper the analysis is performed in a time series context.
} 
although there seem to be no marked differences in the coefficient magnitudes across quantiles. Instead, the shape of the regression quantiles appears to be essentially flat implying the absence of differential effects of technological change. The goodness-of-fit statistics indicate that the power specification is able to explain considerably more than 30 percent of the variation of the $(\log )$ value added shares consistently across all quantiles.

Figure 5

Quantile Regression Results for the Power Specification (Ordinary QR)
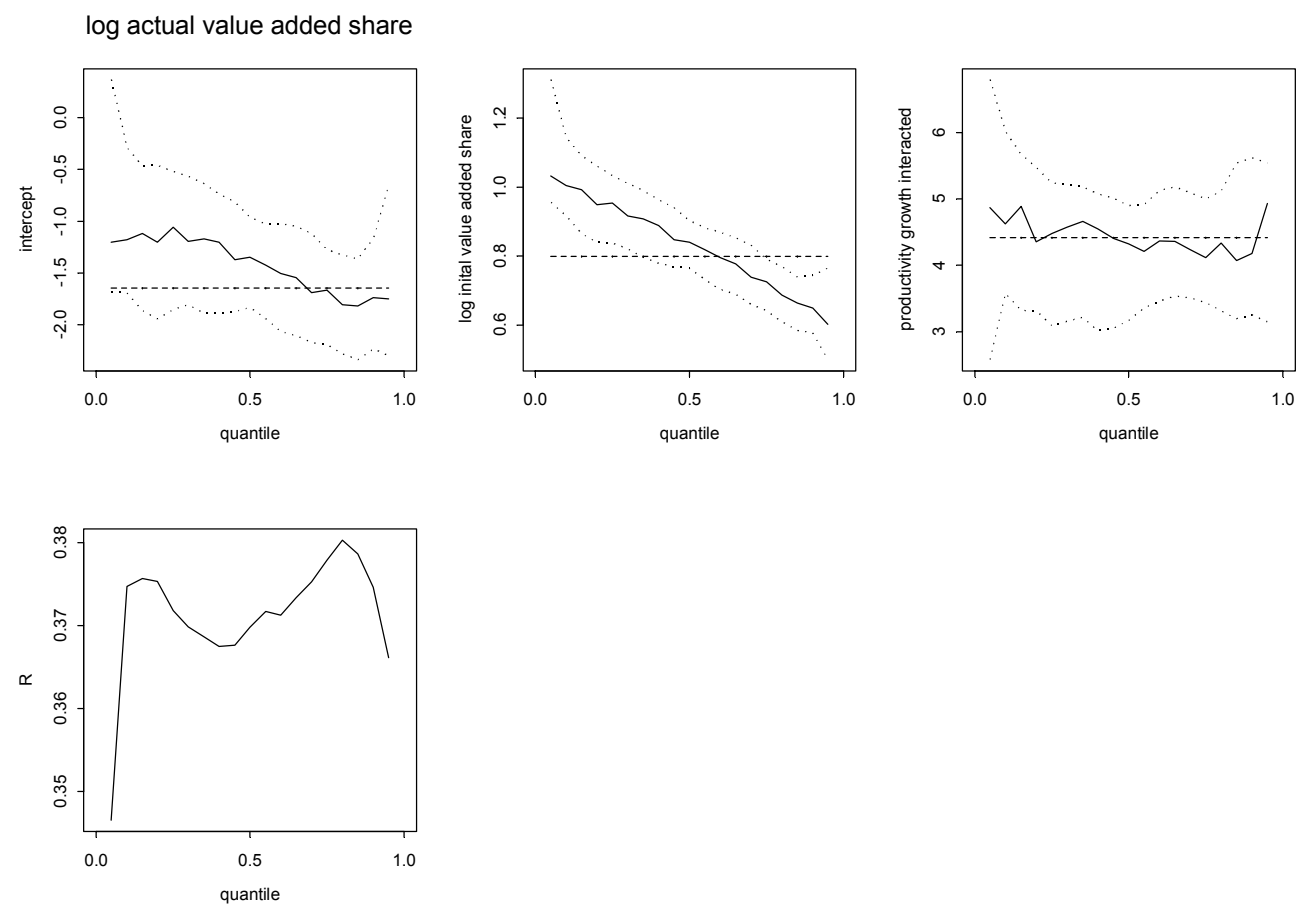

Note that the dotted lines indicate the 95 percent confidence intervals based on 10000 bootstrap replications.

The results for the corresponding instrumental variable quantile regression in figure 6 show compared to the ordinary quantile regression estimates little changes regarding the effect of the initial value added share and the associated persistence interpretation. With respect to productivity growth the general result of a significanly positive influence of this variable is also not affected by the introduction of the instrumental variables. The confidence intervals, however, are noticeably wider now. The fit appears to be slightly lower compared to the ordinary quantile regression estimates. 
Figure 6

Quantile Regression Results for the Power Specification (QR with IV)
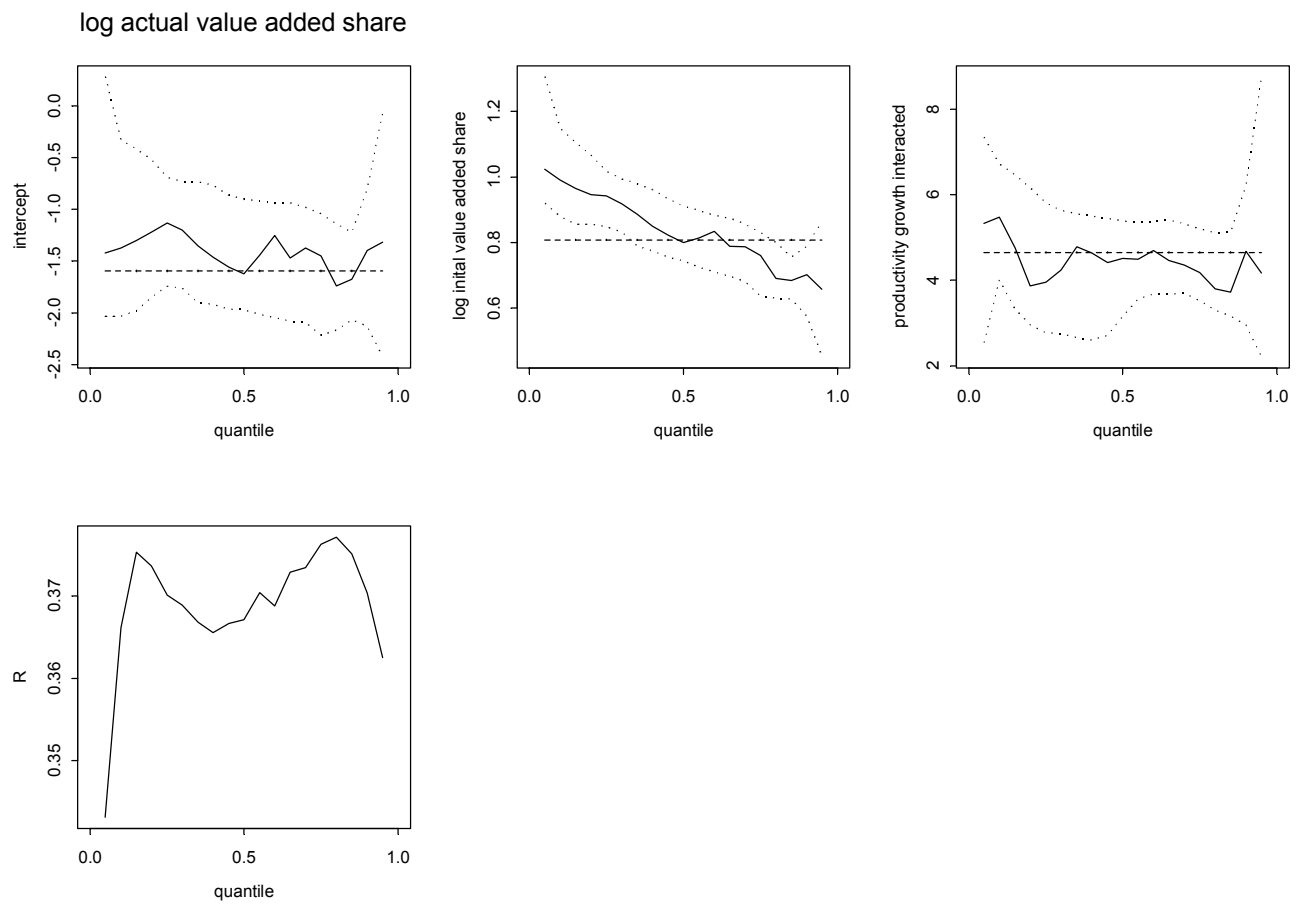

Note that the dotted lines indicate the 95 percent confidence intervals based on 10000 bootstrap replications.

To summarize the results of the quantile regression estimates, the main findings are besides the expected persistence of the share dynamics that productivity change exerts a positive effect on the share dynamics which is statistically significant across all quantiles. Thus, industries with larger productivity growth relative to the leading industry systematically gain in terms of value added shares. Moreover, the efficiency loss often observed in instrumental variables estimations seems to be quite modest in the present case and the overall fit of the quantile regressions is quite acceptable for cross section regressions in industrial organization applications. 


\section{Summary and Conclusions}

The present investigation of structural change within the U.S. manufacturing sector and the investigation of the role of productivity in this process has been entirely conducted in the distribution dynamics framework. We started with a statistical exploration of the value added share dynamics finding a stationary shape of the distribution that is associated with substantial movements within this distribution as revealed by a nonparametric estimate of the transition density. The next step was to construct a model based on a Markov process for the value added shares and then to invoke a search mechanism for innovations that are associated with specific productivity improvements to derive the transition density. This has been done for two different specifications, the power specification in the main text and the logistic specification in the appendix. Although theoretically more appealing (at least on the state-space $[0,1]$ ), the latter specification fits much worse to the data compared to the former specification. The superior empirical fit of the power specification becomes strikingly evident from the maximum likelihood estimate of the transition density of the model which replicates all essential characteristics of the nonparametric fit of the transition density. In addition, the quantile regression estimates show a better fit in the case of the power specification compared to the logistic specification, although the statistical inference regarding persistence and the throughout significantly positive effect of productivity change appear to be the same in both cases.

The implications of these results can be summarized in three points. First, structural change takes place but seems to be bounded in some way, at least during the period 1958-96. The computer revolution leaves a distinct imprint in the transition law of the value added shares, although this is confined only to some of the core industries. The exceptional growth of these industries gives a hint at the enormous growth prospects of these technologies for the rest of the economy. Second, there are differences in the persistence of the value added share dynamics across industries. This points to the existence of asymmetries in the adjustment dynamics of the value added shares as response to shocks. Third, the change of competitiveness in the form of total factor productivity growth relative to the competitors is a variable that exerts a significant influence on the process of structural change. Here again, the effect of the computer revolution and the increasing intensity of computer applications in the other industries may be one of the forces that drive these results. 


\section{Appendix: Logistic Specification}

This specification features the logistic nature of growth processes taken over from biology. As the power function it ensures that the shares are bounded within the interval $[0,1]$ and is furthermore consistent with a unique and stable stationary distribution as shown below. The logistic specification assumes that the value added shares develop according to the law of motion $s_{t}=\left(1+\exp \left(\beta\left(\Delta \tilde{a}_{t}\right)-\beta_{0} s_{t-1}\right)\right)^{-1}$, where $\beta(\cdot)$ now has the properties $\beta(x) \in \mathbb{R}$ and $\beta^{\prime}(x) \leq 0$ for all $x \in \mathbb{R}$ which in part deviate from the power specification. The limiting properties are in this case $\lim _{x \rightarrow \infty} \beta(x)=-\infty$ and $\lim _{x \rightarrow-\infty} \beta(x)=\infty$.

The set $A$ is here given by

$$
A=\left[b\left(\beta_{0} s-\ln \frac{s_{1}}{1-s_{1}}\right), b\left(\beta_{0} s-\ln \frac{s_{2}}{1-s_{2}}\right)\right]
$$

where $b(\cdot)$ denotes again the inverse of $\beta(\cdot)$. In the case of the logistic specification the cdf associated with the transtition kernel is

$$
P\left(s,\left[0, s_{2}\right]\right)=F\left(b\left(\beta_{0} s-\ln \frac{s_{2}}{1-s_{2}}\right)\right)^{\lambda(s)}
$$

since $\lim _{s_{1} \backslash 0} \ln \frac{s_{1}}{1-s_{1}}=-\infty$ and $\lim _{x \rightarrow \infty} b(x)=-\infty$ by the properties of $\beta(\cdot)$.

This transition kernel is increasing in the above defined sense since for $s \geq s^{\prime}$ it follows that $b\left(\beta_{0} s-\ln \frac{s_{2}}{1-s_{2}}\right) \leq b\left(\beta_{0} s^{\prime}-\ln \frac{s_{2}}{1-s_{2}}\right) \quad$ which implies $F\left(b\left(\beta_{0} s-\ln \frac{s_{2}}{1-s_{2}}\right)\right) \leq F\left(b\left(\beta_{0} s^{\prime}-\ln \frac{s_{2}}{1-s_{2}}\right)\right)$ and thus $F\left(b\left(\beta_{0} s-\ln \frac{s_{2}}{1-s_{2}}\right)\right)^{\lambda(s)} \leq F\left(b\left(\beta_{0} s^{\prime}-\ln \frac{s_{2}}{1-s_{2}}\right)\right)^{\lambda\left(s^{\prime}\right)}$.

Here, the MMC condition of Hopenhayn and Prescott (1992) is satisfied for $m=1$ since there exists an $s^{*} \in(0,1)$ such that

$$
P\left(1,\left[0, s^{*}\right]\right)=F\left(b\left(\beta_{0}-\ln \frac{s^{*}}{1-s^{*}}\right)\right)^{\lambda(1)}>0 \forall s^{*} \in(0,1)
$$

and

$$
P\left(0,\left[s^{*}, 1\right]\right)=1-F\left(b\left(-\ln \frac{s^{*}}{1-s^{*}}\right)\right)^{\lambda(0)}>0 \forall s^{*} \in(0,1) .
$$

This establishes the existence and ergodicity of the stationary distribution in the case of the logistic specification. 
The cdf of the truncated Poisson mixture distribution is in this case

$$
P\left(s,\left[0, s_{2}\right]\right)=\frac{\exp \left(\lambda(s) F\left(b\left(\beta_{0} s-\ln \frac{s_{2}}{1-s_{2}}\right)\right)\right)-1}{\exp (\lambda(s))-1}
$$

Assuming $\beta\left(\Delta \tilde{a}_{t}\right)=-\beta_{1} \Delta \tilde{a}_{t}$ leads to $b\left(\beta_{0} s_{t-1}-\ln \frac{s_{t}}{1-s_{t}}\right)=\beta_{1}^{-1}\left(\ln \frac{s_{t}}{1-s_{t}}-\beta_{0} s_{t-1}\right)$ as the inverse function and the respective transition distribution and density functions

$$
\begin{aligned}
P\left(s_{t-1},\left[0, s_{t}\right]\right)= & \frac{\exp \left(\left(\lambda_{0}+\lambda_{1} \ln s_{t-1}\right) \Phi\left(\left(\ln \frac{s_{t}}{1-s_{t}}-\beta_{0} s_{t-1}\right) /\left(a_{1} \beta_{1}\right)-a_{0} / \alpha_{1}\right)\right)-1}{\exp \left(\lambda_{0}+\lambda_{1} \ln s_{t-1}\right)-1} \\
p\left(\ln s_{t} \mid \ln s_{t-1}\right)= & \frac{\exp \left(\left(\lambda_{0}+\lambda_{1} \ln s_{t-1}\right) \Phi\left(\left(\ln \frac{s_{t}}{1-s_{t}}-\beta_{0} s_{t-1}\right) /\left(a_{1} \beta_{1}\right)-a_{0} / a_{1}\right)\right)}{\exp \left(\lambda_{0}+\lambda_{1} \ln s_{t-1}\right)-1} \\
& \cdot\left(\lambda_{0}+\lambda_{1} \ln s_{t-1}\right) \phi\left(\left(\ln \frac{s_{t}}{1-s_{t}}-\beta_{0} s_{t-1}\right) /\left(a_{1} \beta_{1}\right)-a_{0} / a_{1}\right) \\
& \cdot\left(1 /\left(a_{1} \beta_{1}\left(1-s_{t}\right)\right),\right.
\end{aligned}
$$

where the last (Jacobian) factor is again always nonnegative since $s_{t} \in[0,1]$. Plugging the formula for the transition density into the loglikelihood function $\ln L$ as above in the case of the power specification and numerically maximizing this with respect to the same six parameters using the BFGS method gives the transition density depicted in figure 7. 
Figure 7

Transition Density for Logistic Specification (ML Fit)

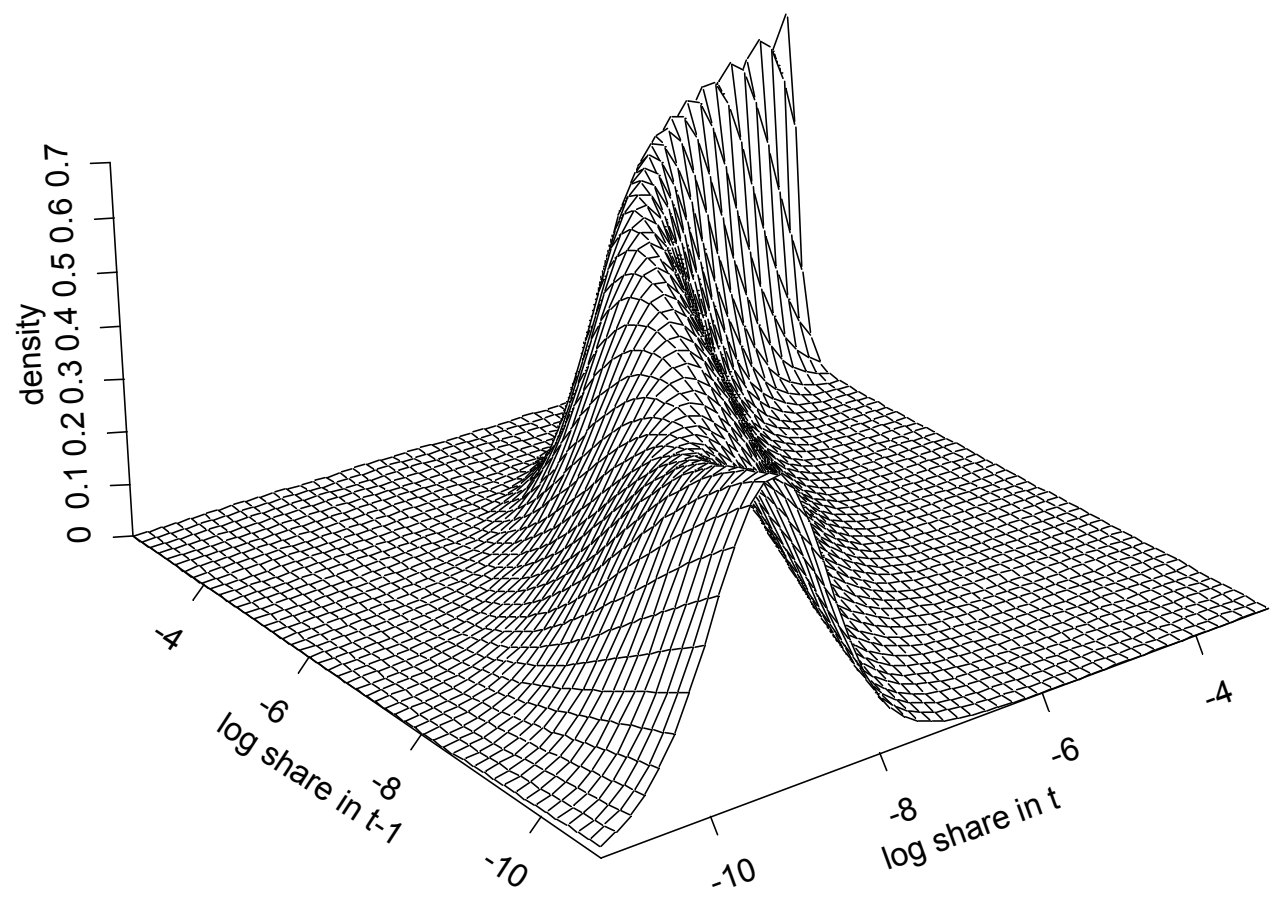

This plot reveals that the differences of the persistence in the case of industries with small and large value added shares are also captured by this transition density. Overall, however, this plot differs considerably from the nonparametric estimate in figure 2 above.

The logistic specification can be transformed to a linear functional estimable by quantile regression by the means of the logit transformation. Point of departure is the logistic function $s_{i t}=\left(1+\exp \left(\beta\left(\Delta \tilde{a}_{i t}\right)-\beta_{0} s_{i t-1}\right)\right)^{-1}$ from which the logit transformation leads to

$$
\ln \left(s_{i t} /\left(1-s_{i t}\right)\right)=\beta_{0} s_{i t-1}-\beta\left(\Delta \tilde{a}_{i t}\right)+u_{i t}=\beta_{0} s_{i t-1}+\beta_{1} \Delta \tilde{a}_{i t}+u_{i t},
$$

where $\beta\left(\Delta \tilde{a}_{i t}\right)=-\beta_{1} \Delta \tilde{a}_{i t}$ in this case.

The ordinary quantile regression results for the logistic specification are reported in figure 8 . They show that the intercept is again significantly negative. The effect of the initial value added share, which appears without logarithm in this specification, is now increasing but only weakly so. The regression quantiles for productivity growth are approximately constant and 
significantly positive for all quantiles. The overall fit of the logistic specification is lower, in particular for the lowest quantiles. This resembles the greater differences of the estimated transtition density of the logistic specification in figure 7 to the nonparametric estimate compared to the respective transtition density of the power specification.

\section{Figure 8}

Quantile Regression Results for the Logistic Specification (Ordinary QR)

logit actual value added share

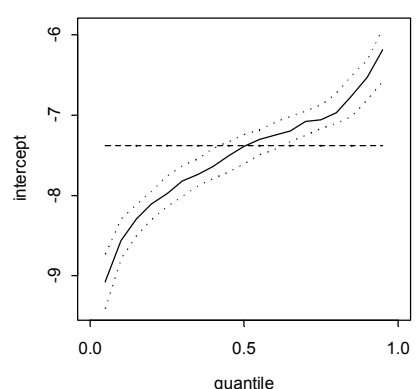

quantile

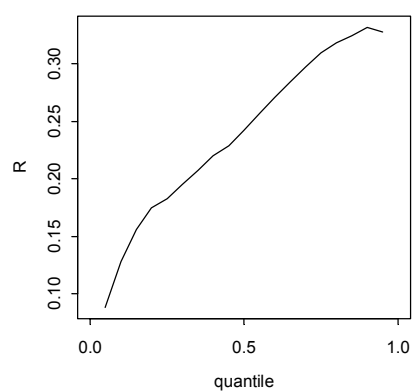

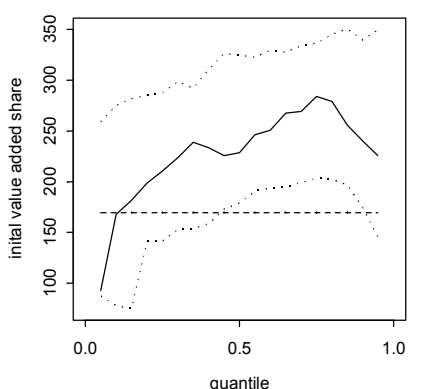

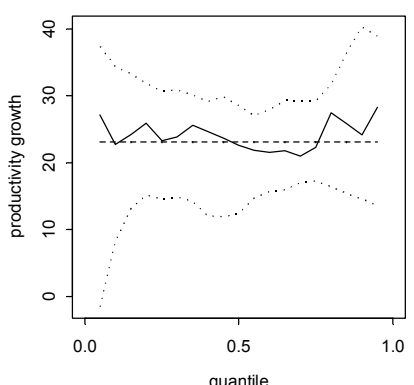

Note that the dotted lines indicate the 95 percent confidence intervals based on 10000 bootstrap replications.

The comparison with the instrumental variable estimates of the logistic specification in figure 9 shows that the main conclusions also hold under these circumstances, although marginally wider confidence intervals can be recognized. 
Figure 9

Quantile Regression Results for the Logistic Specification (QR with IV)

logit actual value added share
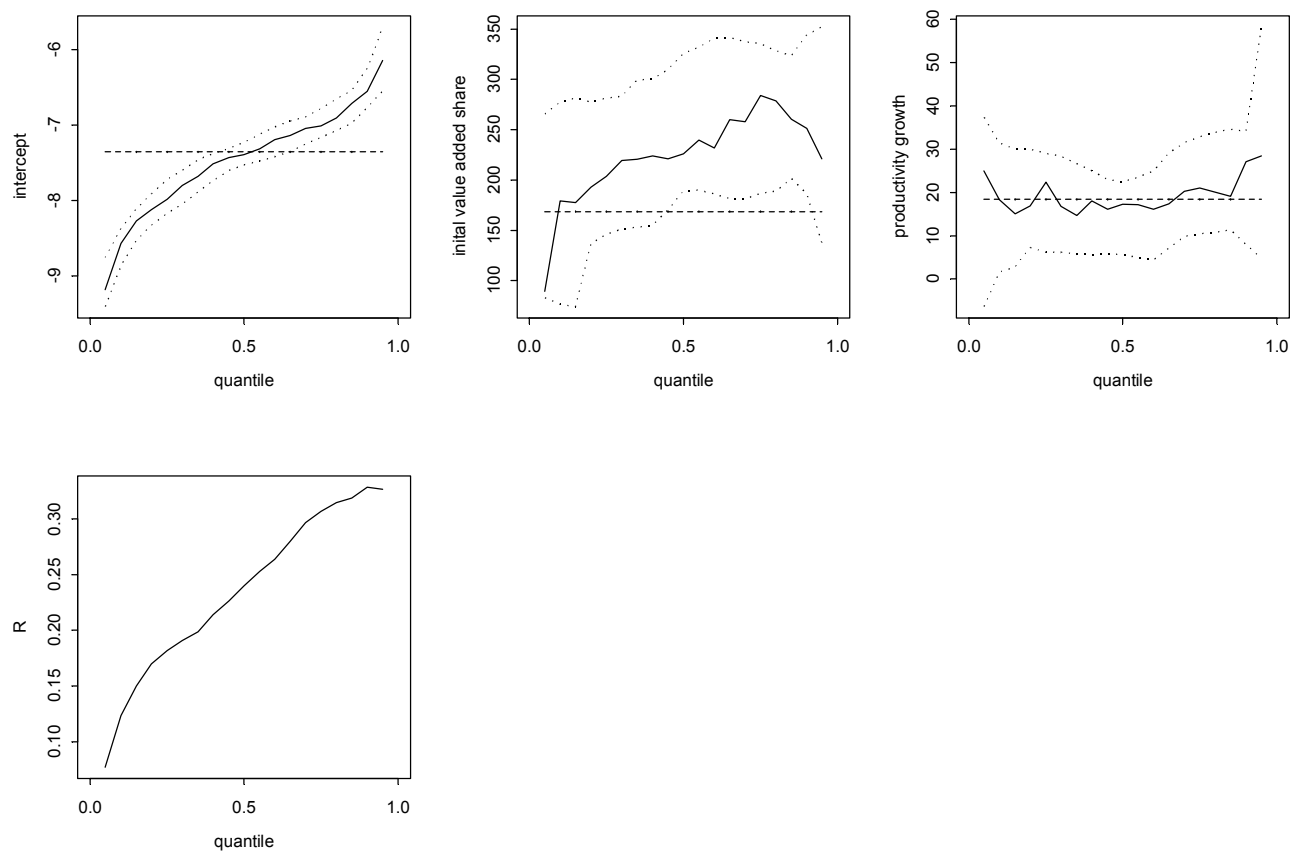

Note that the dotted lines indicate the 95 percent confidence intervals based on 10000 bootstrap replications. 


\section{References}

Andersen, P., Petersen, N.C. (1993), A Procedure for Ranking Efficient Units in Data Envelopment Analysis, Management Science, vol. 39, pp. 1261-1264.

Arias, O., Hallock, K.F., Sosa-Escudero, W. (2001), Individual Heterogeneity in the Returns to Schooling: Instrumental Variables Quantile Regression Using Twins Data, Empirical Economics, vol. 26, pp. 7-40.

Arnold, B.C., Balakrishnan, N., Nagaraja, H.N. (1992), A First Course in Order Statistics, New York: Wiley.

Baily, M.N., Bartelsman, E.J., Haltiwanger, J.C. (1996), Downsizing and Productivity Growth: Myth or Reality?, Small Business Economics, vol. 8, pp. 259-278.

Balk, B.M. (2003), The Residual: On Monitoring and Benchmarking Firms, Industries, and Economies with Respect to Productivity, Journal of Productivity Analysis, vol. 20, pp. 5-47.

Banker, R.D., Gadh, V.M., Gorr, W.L. (1993), A Monte Carlo Comparison of Two Production Frontier Estimation Methods: Corrected Ordinary Least Squares and Data Envelopment Analysis, European Journal of Operational Research, vol. 67, pp. 332-343.

Bartelsman, E.J., Doms, M. (2000), Understanding Productivity: Lessons from Longitudinal Microdata, Journal of Economic Literature, vol. 38, pp. 569-594.

Bartelsman, E.J., Gray, W. (1996), The NBER Manufacturing Productivity Data Base, NBER Technical Working Paper 205.

Basu, S., Fernald, J.G. (1997), Returns to Scale in U.S. Production: Estimates and Implications, Journal of Political Economy, vol. 105, pp. 249-283.

Baumol, W.J. (1967), Macroeconomics of Unbalanced Growth: The Anatomy of Urban Crisis, American Economic Review, vol. 57, pp. 415-426.

Brock, W.A. (1999), Scaling in Economics: A Reader's Guide, Industrial and Corporate Change, vol. 8, pp. 409-446.

Buchinsky, M. (1998), Recent Advances in Quantile Regression Models: A Practical Guide for Empirical Research, Journal of Human Resources, vol. 33, pp. 88-126.

Charnes, A., Cooper, W.W., Lewin, A.Y., Seiford, L.M. (eds.) (1994), Data Envelopment Analysis: Theory, Methodology, and Application, Boston: Kluwer Academic Publishers.

Chen, L.A., Portnoy, S. (1996), Two-Staged Regression Quantiles and Two-Staged Trimmed Least-Squares Estimators for Structural Equation Models, Communication in Statistics: Theory and Methods, vol. 25, pp. 1005-1032.

Davidson, R., MacKinnon, J.G. (2003), Econometric Theory and Methods, Oxford: Oxford University Press. 
Dennis, J.E., Schnabel, R.B. (1983), Numerical Methods for Unconstrained Optimization and Nonlinear Equations, Prentice-Hall, Englewood Cliffs.

Färe, R., Grosskopf, S., Norris, M., Zhang, Z. (1994), Productivity Growth, Technical Progress, and Efficiency Change in Industrialized Countries, American Economic Review, vol. 84, pp. 66-83.

Färe, R., Grosskopf, S., Roos, P. (1998), Malmquist Productivity Indexes: A Survey of Theory and Practice, in: R. Färe, S. Grosskopf, R.R. Russell (eds.), Index Numbers: Essays in Honour of Sten Malmquist, Boston: Kluwer, pp. 127-190.

Fitzenberger, B., Koenker, R.W., Machado, J.A.F. (2001), Introduction, Empirical Economics, vol. 26, pp. 1-5.

Greene, W.H. (1993), The Econometric Approach to Efficiency Analysis, in: H.O. Fried, C.A.K. Lovell, S.S. Schmidt (eds.), The Measurement of Productive Efficiency, Oxford: Oxford University Press, pp. 68-119.

Hahn, J. (1995), Bootstrapping Quantile Regression Estimators, Econometric Theory, vol. 11, pp. 105-121.

Harberger, A.C. (1998), A Vision of the Growth Process, American Economic Review, vol. 88 , pp. 1-32.

Hopenhayn, H.A., Prescott, E.C. (1992), Stochastic Monotonicity and Stationary Distributions for Dynamic Economies, Econometrica, vol. 60, pp. 1387-1406.

Inada, K.-I. (1963), On a Two-Sector Model of Economic Growth: Comments and a Generalization, Review of Economic Studies, vol. 30, pp. 119-127.

Jones, M.C., Marron, J.S., Sheather, S.J. (1996), A Brief Survey of Bandwidth Selection for Density Estimation, Journal of the American Statistical Association, vol. 91, pp. 401-407.

Koenker, R.W. (1994), Confidence Intervals for Regression Quantiles, in: P. Mandl, M. Hušková (eds.), Asymptotic Statistics, New York: Springer, pp. 349-359.

Koenker, R.W., (2000), Quantile Regression, article prepared for the statistics section of the International Encyclopedia of the Social Sciences.

Koenker, R.W., Bassett, G. (1978), Regression Quantiles, Econometrica, vol. 46, pp. 33-50.

Koenker, R.W., Hallock, K.F. (2001), Quantile Regression, Journal of Economic Perspectives, vol. 15, pp. 143-156.

Koenker, R.W., Machado, J.A.F. (1999), Goodness of Fit and Related Inference Procedures for Quantile Regression, Journal of the American Statistical Association, vol. 94, pp. 1296-1310.

Koenker, R.W., Xiao, Z. (2004), Unit Root Quantile Autoregression Inference, Journal of the American Statistical Association, forthcoming.

Kongsamut, P., Rebelo, S., Xie, D. (2001), Beyond Balanced Growth, Review of Economic Studies, vol. 68, pp. 869-882. 
Krüger, J.J. (2005), Productivity Dynamics Beyond-the-Mean in U.S. Manufacturing Industries: An Application of Quantile Regression, Empirical Economics, forthcoming 2005 .

Kumar, S., Russell, R.R. (2002), Technological Change, Technological Catch-up, and Capital Deepening: Relative Contributions to Growth and Convergence, American Economic Review, vol. 92, pp. 527-548.

Kuznets, S. (1966), Modern Economic Growth: Rate, Structure, and Spread, New Haven: Yale University Press.

Lipsey, R.G., Carlaw, K. (2000), What Does Total Factor Productivity Measure?, International Productivity Monitor, no. 1, pp. 31-40.

Loury, G.C. (1981), Intergenerational Transfers and the Distribution of Earnings, Econometrica, vol. 49, pp. 843-867.

Meyn, S.P., Tweedie, R.L. (1993), Markov Chains and Stochastic Stability, New York: Springer.

Quah, D. (1996a), Convergence Empirics Across Economies with (Some) Capital Mobility, Journal of Economic Growth, vol. 1, pp. 95-124.

Quah, D. (1996b), Twin Peaks: Growth and Convergence in Models of Distribution Dynamics, Economic Journal, vol. 106, pp. 1045-1055.

Ruggiero, J. (1999), Efficiency Estimation and Error Decomposition in the Stochastic Frontier Model: A Monte Carlo Analysis, European Journal of Operational Research, vol. 115 , pp. 555-563.

Sheather, S.J., Jones, M.C. (1991), A Reliable Data-Based Bandwidth Selection Method for Kernel Density Estimation, Journal of the Royal Statistical Society, Series B, vol. 53, pp. 683-690.

Solow, R.M. (1957), Technical Change and the Aggregate Production Function, Review of Economics and Statistics, vol. 39, pp. 312-320.

Stokey, N.L., Lucas, R.E. with E.C. Prescott (1989), Recursive Methods in Economic Dynamics (with Edward Prescott), Cambridge (Mass.): Harvard University Press.

Sutton, J. (1997), Gibrat's Legacy, Journal of Economic Literature, vol. 35, pp. 40-59.

Tweedie, R.L. (2001), Markov Chains: Structure and Applications, in: D.N. Shanbhag, C.R. Rao (eds.), Handbook of Statistics, vol. 19, Amsterdam: Elsevier, pp. 817-851.

Wand, M.P., Jones, M.C. (1995), Kernel Smoothing, London: Chapman\&Hall.

Wolfstätter, E. (1999), Topics in Microeconomics: Industrial Organization, Auctions, and Incentives, Cambridge (Mass.):Cambridge University Press. 